\title{
Spinorial Path Integral for Loop Gravity: Coherent states and Spinfoam symmetries
}

\author{
Etera Livine* \\ ENS Lyon - Laboratoire de Physique - CNRS UMR 5672 \\ E-mail: etera.livinedens-lyon.fr
}

\begin{abstract}
The spinfoam framework defines transition amplitudes for spin network states of loop quantum gravity. I will review their recent reformulation in terms of spinorial variables allowing to see these amplitudes as coherent state path integrals [1,2,3]. This clarifies their geometrical meaning and at the identification of symmetries: recursion relations for $3 \mathrm{nj}$ symbols are turned into Hamiltonian constraints satisfied by the spinfoam amplitudes [4,5]. This applies in particular to the derivation of modified FRW equations for quantum cosmology [6].

References

[1] L Freidel and S Speziale, From twistors to twisted geometries, Phys. Rev. D 82, 084041 (2010) arXiv:1006.0199 [gr-qc]

[2] E F Borja, L Freidel, I Garay and E R Livine, U(N) tools for Loop Quantum Gravity: The Return of the Spinor, Class. Quant. Grav. 28, 055005 (2011) arXiv:1010.5451 [gr-qc]

[3] M Dupuis and E R Livine, Holomorphic Simplicity Constraints for 4d Riemannian Spinfoam Models, J. Phys. Conf. Ser. 360, 012046 (2012) arXiv:1111.1125 [gr-qc]

[4] V Bonzom, E R Livine and S Speziale, Recurrence relations for spin foam vertices, Class. Quant. Grav. 27, 125002 (2010) arXiv:0911.2204 [gr-qc]

[5] V Bonzom and E R Livine, Generating Functions for Coherent Intertwiners, Class. Quant. Grav. 30, 055018 (2013) arXiv:1205.5677 [gr-qc]

[6] E R Livine and M Martin-Benito, Classical Setting and Effective Dynamics for Spinfoam Cosmology, Class. Quant. Grav. 30, 035006 (2013) arXiv:1111.2867 [gr-qc]
\end{abstract}

Frontiers of Fundamental Physics 14 - FFP14,

15-18 July 2014

Aix Marseille University (AMU) Saint-Charles Campus, Marseille

* Speaker. 